\title{
THE OUTBURST OF CI CYGNI IN 1973
}

\author{
T. S. BELYAKINA
}

Crimean Astrophysical Observatory, U.S.S.R.

\begin{abstract}
Description of photometric and spectroscopic observations of CI Cyg during the 1973 outburst.
\end{abstract}

Several symbiotic stars have periodic light-curves. CI Cyg is one of those stars. Photographic observations of CI Cyg made from 1890 to 1967 have shown light variations with a period equal to 855 days and a mean amplitude of about $1^{m}\left(m_{\mathrm{pg}} \sim 12^{\mathrm{m}}-\right.$ $13^{m}$ ) (Aller, 1954; Greenstein, 1973; Hoffleit, 1968). Two outbursts were noticed in 1911 and in 1937 when the brightness of the star reached $m_{\mathrm{pg}}=10^{m} \cdot 7$ and $m_{\mathrm{pg}}=10^{m} \cdot 2$, respectively (Greenstein, 1937; Hoffleit, 1968). The duration of each outburst was about 200 days.

The activity of CI Cyg increased at the beginning of the seventies. Two outbursts have appeared during 1971-73 (Belyakina, 1974; Hoffleit, 1971; Lowder, 1971; Mayall, 1973; Stienon, 1973; Swings and Allen, 1972).

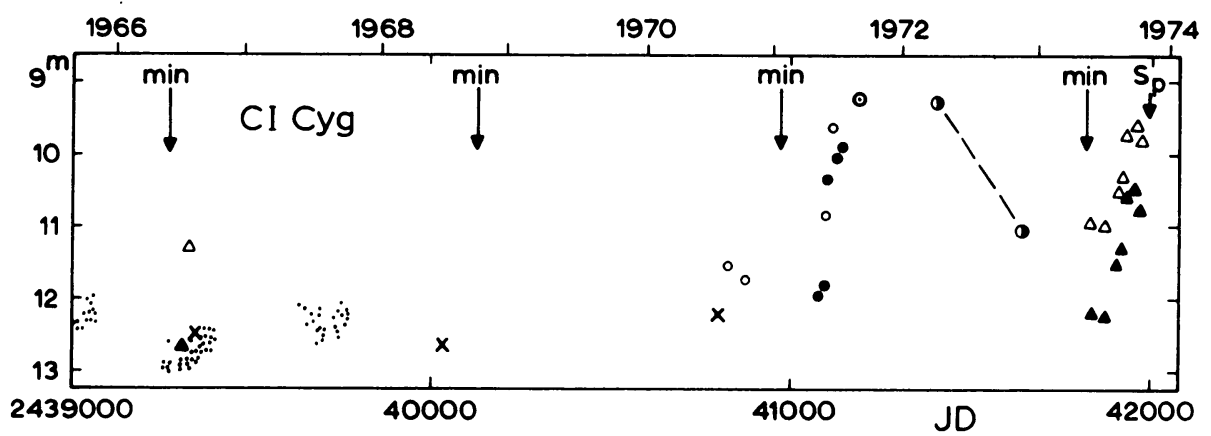

Fig. 1. The light variations of CI Cyg in 1966-73.

$$
\begin{array}{ll}
\cdot-m_{\mathrm{pg}} \text { (Hoffleit, 1968) } & \bigcirc-m_{v} \text { (Swings and Allen, 1972) } \\
\times-m_{\mathrm{pg}} \text { (Belyakina, here) } & -m_{v} \text { (Mayall, 1973) } \\
-m_{\mathrm{pg}} \text { (Hoffleit, 1971) } & \triangle-V \text { (Belyakina, 1967, 1974) } \\
\bigcirc-m_{v} \text { (Lowder, 1971) } & \Delta-B \text { (Belyakina, 1967, 1974) }
\end{array}
$$

The arrows indicate minima of the composite light curve of $\mathrm{Cl}$ Cyg and the short arrow indicates the date of the spectrographic observations.

All.published photographic, photoelectric and visual estimates of the stellar brightness carried out in 1966-73 by different observers are collected in Figure 1 (Belyakina, 1967, 1974; Hoffleit, 1968, 1971; Mayall, 1973; Lowder, 1971; Swings and Allen, 1972). Figure 1 shows that the brightness of CI Cyg reached $m_{\mathrm{pg}}=9^{m} .9$ in 1971 and $m_{\mathrm{pg}}=10^{m} .5$ in 1973. The duration of the outburst in 1971-72 was about 500 days. The outburst in 1973 lasted less than 350 days as the brightness of CI Cyg in June, 1974 was close to the usual. 
Our photoelectric observations of CI Cyg were plotted on a two-colour diagram (see Figure 2). It follows from Figure 2 that the colour variations of the variable may be explained by assuming that the star is a binary system consisting of a cold M2III star and a hot star $\left(T_{\mathrm{e}}=5 \times 10^{4} \mathrm{~K}\right)$ surrounded by a gaseous nebula. The outburst of CI Cyg in 1973 was caused by the increasing in brightness of the hot component by about $1^{m}$ in the visual.

Several spectrograms, covering three regions of the CI Cyg spectrum, were taken by K. K. Chuvaev with an image-tube spectrograph at the $2.6-\mathrm{m}$ telescope of the Crimean observatory. The mean dispersion was about $40 \AA \mathrm{mm}^{-1}$. The observations were carried out in 1973, October 30 , two weeks after the maximum brightness. The detected emission lines and their equivalent widths are shown in Table $\mathrm{I}$.

One can see that the [O III] line at $5007 \AA$ was clearly visible in 1973, while in 1948, 1952, 1955 and 1964 (Aller, 1957; Boyarchuk, 1969; Merrill, 1950; Tcheng Mao-Lin and Bloch, 1954) it was not seen in the stellar spectrum at all.

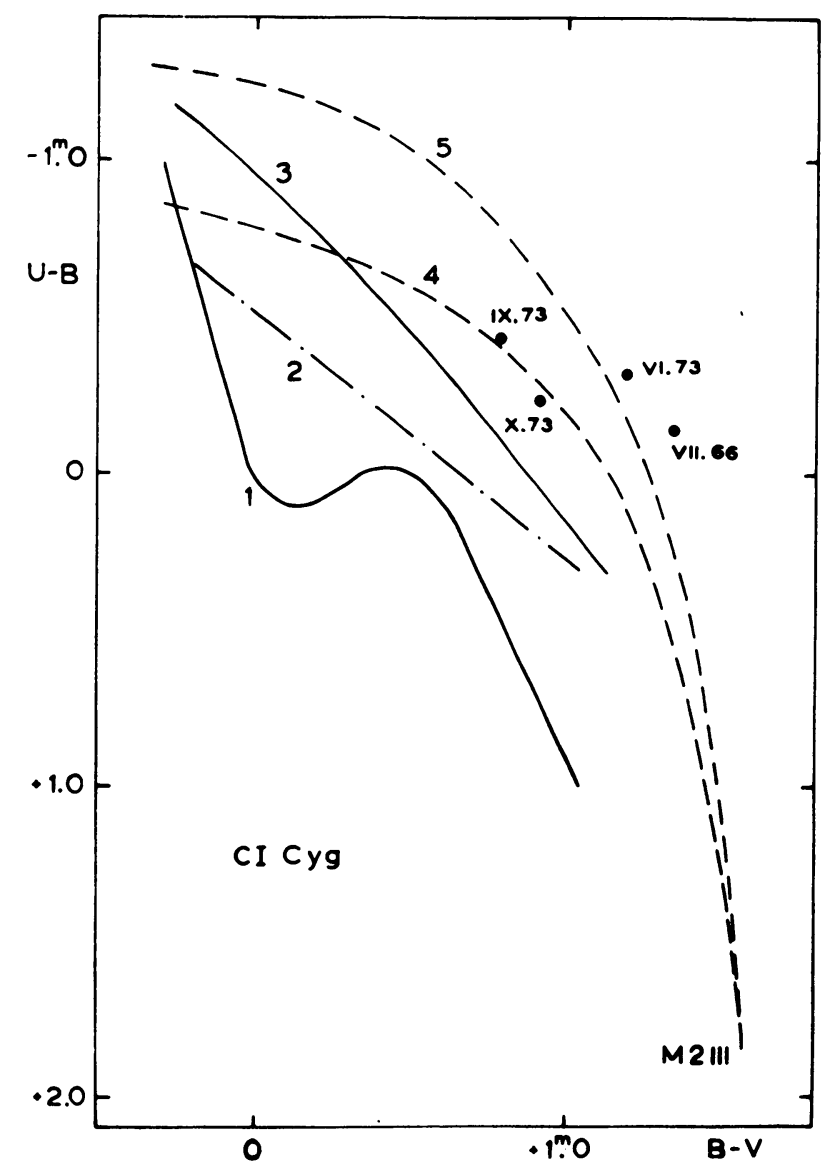

Fig. 2. The colour variations of CI Cyg. 1 - main sequence line; 2 - reddening line; 3 - black-body line; 4 - composite radiation line : cold star $\mathrm{M} 2 \mathrm{III}+\operatorname{gas}\left(T_{\mathrm{e}}=17000 \mathrm{~K}, n_{\mathrm{e}} \geqslant 10^{6} \mathrm{~cm}^{-3}\right)$; and 5 - composite radiation line: cold star M2III +hot $\operatorname{star}\left(T_{\mathrm{e}}=5 \times 10^{4} \mathrm{~K}\right)$ 
TABLE I

The equivalent widths of $\mathrm{CI}$ Cyg emission lines

\begin{tabular}{lllr}
\hline Spectral region $(\AA)$ & Element & $\lambda(\AA)$ & $\mathrm{W}_{\lambda}(\AA)$ \\
\cline { 3 - 4 } & & & \\
& He II & 4866 & 7.5 \\
$4655-5200$ & He I & 4713 & 2.5 \\
& H $\beta$ & 4861 & 58.5 \\
& He I & 4922 & 5.4 \\
$5550-6050$ & {$\left[O_{\text {III }}\right]$} & 5007 & 6.8 \\
$6450-6700$ & He I & 5016 & 3.3 \\
& He I & 5876 & 10.4 \\
& H $\alpha$ & 6563 & 160 \\
& He I & 6678 & 5 \\
\hline
\end{tabular}

\section{References}

Aller, L. H.: 1954, Publ. Dominion Astrophys. Obs. 9, 343.

Aller, L. H.: 1957, Mem. Soc. Roy. Sci. Liège 18, fasc. 1, 305.

Belyakina, T. S.: 1967, Izv. Krymsk. Astrofiz. Obs. 38, 171.

Belyakina, T. S.: 1974, Info. Bull. Var. Stars, No. 863.

Boyarchuk, A. A.: 1969, Izv. Krymsk. Astrofiz. Obs. 39, 124.

Boyarchuk, A. A.: 1970, in A. A. Boyarchuk and R. E. Gershberg (eds.), Eruptive Stars, Nauka, Moscow, p. 149. (In Russian.)

Greenstein, N. K. G.: 1937, Harvard Bull., No. 906.

Hoffleit, D.: 1968, Irish Astron. J. 8, 149.

Hoffleit, D.: 1971, IAU Circ. No. 2336.

Lowder, W. M.: 1971, IAU Circ. No. 2335.

Mayall, M. W.: 1973, J. Roy. Astron. Soc. Canada 67, 101.

Merrill, P. V.: 1950, Astrophys. J. 111, 484.

Stienon, F. M.: 1973, Bull. Amer. Astrophys. Soc. 5, 17.

Swings, J. P. and Allen, D. A.: 1972, Publ. Astron. Soc. Pacific 84, 523.

Tcheng Mao-Lin and Bloch, M.: 1954, Ann. Astrophys. 17, 6.

\section{DISCUSSION}

A. S. Sharov: Does narrow-band photometry exist of these stars?

T. S. Belyakina: No. 